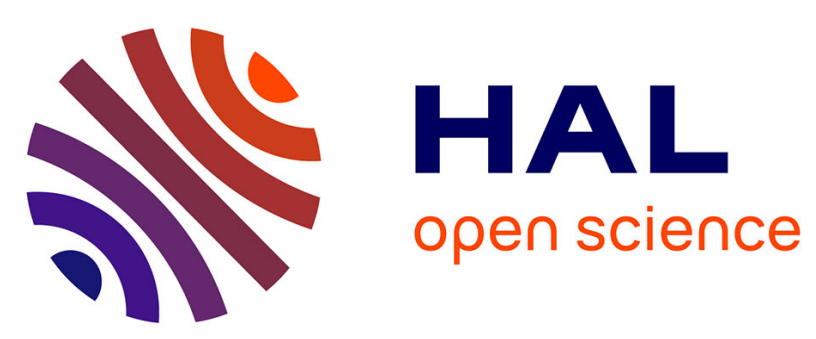

\title{
MODULATION OF SCALE-FREE PROPERTIES OF BRAIN ACTIVITY IN MEG
}

Nicolas Zilber, Philippe Ciuciu, Patrice Abry, Virginie van Wassenhove

\section{To cite this version:}

Nicolas Zilber, Philippe Ciuciu, Patrice Abry, Virginie van Wassenhove. MODULATION OF SCALEFREE PROPERTIES OF BRAIN ACTIVITY IN MEG. IEEE International Symposium on Biomedical Imaging, May 2012, Barcelone, Spain. pp.1531-1534. cea-00699905

\section{HAL Id: cea-00699905 https://hal-cea.archives-ouvertes.fr/cea-00699905}

Submitted on 21 May 2012

HAL is a multi-disciplinary open access archive for the deposit and dissemination of scientific research documents, whether they are published or not. The documents may come from teaching and research institutions in France or abroad, or from public or private research centers.
L'archive ouverte pluridisciplinaire HAL, est destinée au dépôt et à la diffusion de documents scientifiques de niveau recherche, publiés ou non, émanant des établissements d'enseignement et de recherche français ou étrangers, des laboratoires publics ou privés. 


\title{
MODULATION OF SCALE-FREE PROPERTIES OF BRAIN ACTIVITY IN MEG
}

\author{
Nicolas Zilber ${ }^{(1)}$, Philippe Ciuciu ${ }^{(1)}$, Patrice Abry ${ }^{(2)}$ and Virginie van Wassenhove ${ }^{(1,3)}$ \\ ${ }^{(1)}$ CEA/DSV/I² BM NeuroSpin center, Bât. 145, F-91191 Gif-sur-Yvette, France \\ (2) CNRS, UMR 5672, Laboratoire de Physique. ENS Lyon, France. \\ (3) INSERM, U992, Cognitive Neuroimaging Unit, F-91191 Gif-sur-Yvette, France. \\ 1,3 firstname.lastnamedcea.fr, ${ }^{2}$ patrice.abrydens-lyon.fr
}

\begin{abstract}
The analysis of scale-free (i.e., $1 / f$ power spectrum) brain activity has emerged in the last decade since it has been shown that low frequency fluctuations interact with oscillatory activity in electrophysiology, noticeably when exogenous factors (stimuli, task) are delivered to the human brain. However, there are some major difficulties in measuring scale-free activity in neuroimaging data: they are noisy, possibly nonstationary ... Here, we make use of multifractal analysis to better understand the biological meaning of scale-free activity recorded with Magnetoencephalography (MEG) data. On a cohort of 20 subjects, we demonstrate the presence of self-similarity on all sensors during rest and visually evoked activity. Also, we report significant multifractality on the norm of gradiometers. Finally, on the latter signals we show how self-similarity and multifractality are modulated between ongoing and evoked activity.
\end{abstract}

Index Terms - MEG, scale-free activity, scaling, multifractal analysis

\section{INTRODUCTION}

Starting with the discovery of brain oscillations in electroencephalography (EEG) and later in magnetoencephalography (MEG),many studies have attempted to explain their mechanism and their possible functional role in behavior and cognition. Some frequency bands were particularly conspicuous and were given names such as $\delta$ (1$3 \mathrm{~Hz}), \theta(4-8 \mathrm{~Hz}), \alpha(8-12 \mathrm{~Hz}), \beta(12-30 \mathrm{~Hz})$ and $\gamma(>40 \mathrm{~Hz})$. On the other hand, very low frequency activity $(<1 \mathrm{~Hz})$ (also named "infraslow") did not attract as much attention due to the power-law distribution of the spectral density in the range of low frequencies. This property is very ubiquitous in dynamic systems (fully developed turbulence, internet traffic, earthquakes, stock market exchange, ...) [1]. In the context of brain imaging, such activity was initially attributed to the intrinsic $1 / f$ electronic device noise and was systematically removed by high-pass filtering or normalization of M/EEG data.

However, several authors have shown that ongoing activity, the major part of brain activity [2,3], has scale-free dynamics -i.e. a $1 / f$ power spectrum $[1,3-5]$. Since then, the study of scale-free properties has emerged as a new research topic in neuroscience and brain neuroimaging. A major finding is that scale-free activity recorded with EEG interacts with ongoing oscillatory activity [6]. Moreover, the surprising correlation between EEG microstates and fMRI resting-state networks (RSN) could be explained by the scale-free properties of these microstates [7]. Other experiments have shed light on the modulation of scale-free activity by exogenous (drugs,task) and endogenous (brain state, pathology) factors $[1,8,9]$.

Nonetheless, some major difficulties remain when assessing scalefree properties in neuroimaging data: they are noisy, multivariate, nonstationary, and possibly corrupted by physiological artifacts (eye movements, heartbeats...). In particular, the nonstationarity and the long range dependence yield a lack of statistical consistency when estimating scaling properties in the time or frequency domain using methods like Detrended Fluctuation Analysis (DFA) [11]. To overcome this difficulty, one solution is to use wavelet-based analysis of scaling properties.

Wavelet analysis implies a major change of paradigm: frequency (and hence the frequency shift operator) is no longer considered as the relevant descriptor and is replaced instead by scale (and hence by a dilation, or scale change operator). This enables to correctly estimate scale invariance parameters even when data present various forms of non-stationarity. Moreover, some empirical results [5] suggest that ongoing brain activity may be better modeled by the class of multifractal (MF) processes. This class contains and extends that of self similar processes (e.g. fractional Brownian motion, $\mathrm{fBm}$ ) thereby offering a more versatile and richer description of the data in terms of scaling properties and departures from Gaussian property.

In Section 2, we summarize spectral and multifractal analyses. In Section 3, we show the presence of scaling in MEG data at the sensor level and compare standard spectral estimators with the waveletleader based multifractal (WLMF) analysis [10]. Additionally, we study the modulation of multifractal attributes between two cognitive states: i.) Rest (R), and ii.) visualization of Random Dots Kinematograms (RDK). Although no significant difference could be found when analyzing direction-specific gradio- or magneto-meters (see Section4.1), statistical differences are exhibited when analyzing the norm of gradiometers (see Section 4.2). A criterion is derived in Section 4.3 to explain this surprising difference and an interpretation is suggested. Conclusions are drawn in Section 5.

\section{SCALING ANALYSIS}

\subsection{Spectral analysis}

Scaling is classically analysed using standard spectrum estimation such as windowed-averaged periodograms (e.g. Welch's estimator) or detrended fluctuation analysis (DFA) [11]. The information is supposed to be mainly contained in the power spectrum $\Gamma_{X}(f)$ of the signal $X$ for $f \rightarrow 0$, which behaves as a power law:

$$
\Gamma_{\text {Welch }}(f)=\frac{1}{n} \sum_{k=1}^{n} \hat{\Gamma}_{X}\left(f, t_{k}\right) \simeq C|f|^{-\gamma}, \gamma>0 .
$$

This implicitly requires the data to be stationary. Accordingly, fractional Gaussian noise (fGn) is used as the paradigm model for such analysis if $\gamma \in(-1,1)$. However, on real neuroimaging data, the measured exponent $\gamma$ usually satisfies $\gamma \in(1,3)$ [1], the data are thus better modelled by the non-stationary fBm process, whose in- 
crements follow a fGn. For both fBm and fGn, $H$ takes values in the range of $(0,1)$, hence corresponding to $\gamma=2 H+1$, for the former and to $\gamma=2 H-1$ for the latter. However, if analysed data follows a fGn with $H>0.5$, the underlying process is said long range dependent, meaning that its autocorrelation function decreases very slowly, while if the analysed time series follows a fBm and $H>0.5$, the corresponding process is self-similar, hence nonstationary.

The $\mathrm{fBm} / \mathrm{fGn}$ pair is intrinsically coupled to Gaussian processes. However, empirical results on brain imaging data [12] have shown that data often departs from Gaussian distribution, hence ruling out these models. Also, empirical evidence on brain electrical field [1] indicates that the estimated $\gamma$ takes values oscillating around the $\mathrm{fBm} / \mathrm{fGn} \gamma=1$ limit, hence suggesting a constant switch between nonstationary selfsimilarity and stationary long memory, a very unsatisfactory situation for data modeling and interpretation. By using wavelet transforms, the nonstationarity issue can be overcome. Furthermore, the wavelet-based scaling properties (eg, Hurst parameter) are known to be more accurately estimated. Last but not least, departure from Gaussianity can be captured by analyzing statistical properties of the wavelet coefficients at different statistical orders $(\neq 2)$ and interpreting such results in the context of multifractal processes.

\subsection{Multifractal Analysis}

Let $d_{X}(j, k)$ denote the discrete wavelet transform coefficients of a signal $X$, where $j$ denotes the analysis scale $\left(a=2^{j}\right)$ and $k$ the time position $\left(t=2^{j} k\right)$, computed from a Daubechies mother-wavelet $\psi_{0}(t)$. Scale invariance is now commonly and operationally defined as the power law behaviors of the (time average of the $q$-th power of the) $d_{X}(j, k)$, with respect to the analysis scale $j$, for a given (large) range of scales $j \in\left[j_{m}, j_{M}\right], j_{M}>j_{m}$ :

$$
S^{d}(j, q) \triangleq \frac{1}{n_{j}} \sum_{k=1}^{n_{j}}\left|d_{X}(j, k)\right|^{q} \simeq c_{q} 2^{j \zeta^{d}(q)} .
$$

$S^{d}(j, q)$ is the structure function at the scale $j$ and order $q . \zeta^{d}(q)$ refers to the scaling exponents. If $X$ is monofractal (or self-similar), $\zeta^{d}(q)$ is linear and can be simply characterized by one parameter (namely, the Hurst exponent $H$ ). However, if $X$ is multifractal, $\zeta^{d}(q)$ is a concave function and its description is more complex. It is worth noting that the only use of the power spectrum (i.e. $S^{d}(j, q)$ for $q=2$ ) does not give any information on the multifractal properties. Ideally, we should assess $\zeta^{d}(q)$ for all values of $q$ (positive and negative). It has been shown recently that replacing wavelet coefficients $d_{X}(j, k)$ by the so-called wavelet Leaders $L_{X}(j, k)$ would improve the estimation of $\zeta^{d}(q)$, especially for negative values of $q$.

Wavelet Leaders $L_{X}(j, k)$ are defined as the local suprema of wavelet coefficients within a local neighborhood, and over all finer scales: [10]: $L_{X}(j, k)=\sup _{\lambda_{j^{\prime}, k^{\prime}} \subset 3 \lambda_{j, k}}\left|d_{X}\left(j^{\prime}, k^{\prime}\right)\right|$, where $\lambda_{j, k}=$ $\left[k 2^{j},(k+1) 2^{j}\right)$ and $3 \lambda_{j, k}=\cup_{m\{-1,0,1\}} \lambda_{j, k+m}$. Eq. (2) still holds by replacing $\left|d_{X}(j, k)\right|^{q}$ with $L_{X}(j, k)^{q}$ and $\zeta^{d}(q)$ with $\zeta^{L}(q)$. In practice, estimating of $\zeta^{L}(q)$ for all values of $q$ turns out very difficult. We make use of a polynomial expansion of $\zeta^{L}(q)$ instead:

$$
\zeta^{L}(q)=\sum_{p \geq 1} c_{p}^{L} q^{p} / p !=c_{1}^{L} q+c_{2}^{L} \frac{q^{2}}{2}+c_{3}^{L} \frac{q^{3}}{6}+\cdots .
$$

As detailed in [10], the $c_{p}^{L}$ coefficients are estimated by linear regressions, where $c_{1}^{L}$ reflects the self-similarity (or long memory) of the process, especially for monofractal processes, where $c_{1}^{L}=H$ and $\forall p \geq 2 c_{p}^{L}=0$. For multifractal processes, we specifically have $c_{2}^{L}<0$ to quantify the amount of singularities and $c_{3}^{L} \in \mathbb{R}$ for describing the assymetric nature of the singularity distribution. In this study, we have focused on $c_{1}^{L}$ and $c_{2}^{L}$.
The WLBMF toolbox has been designed to robustly estimate these $c_{p}^{L}$ parameters [10]. it has been already used successfully to analyse multifractal properties of fMRI signals [5] and scale-free dynamics in EEG microstates [7]. In the following, it allows us to accurately characterize scale-free properties of MEG time series and their modulation by external stimulus.

\section{POWER LAW SPECTRUM ON MEG RECORDINGS}

\subsection{Data acquisition}

Brain magnetic fields were recorded in a magnetically shielded room using the whole-head Elekta Neuromag MEG system (Elekta LTD, Helsinki) with 102 triple-sensor elements (two orthogonal planar gradiometers and one magnetometer) in upright position. Data were sampled at $2000 \mathrm{~Hz}$ and pass-band filtered between $0.03 \mathrm{~Hz}$ and $600 \mathrm{~Hz}$. Signal Space Separation (SSS) was carried out with Max-Filter (Elekta LTD) to remove external interferences. Occular and cardiac artifacts were removed using principal component analysis based on the recorded electrooculograms (EOG) and electrocardiograms (ECG).

Twenty healthy right-handed subjects (aged 19-27 years) with normal or corrected-to-normal vision participated in this experiment. Each participant provided an informed consent in accordance with the Declaration of Helsinki (2008) and the Ethics Committee on $\mathrm{Hu}-$ man Research at NeuroSpin (Gif-sur-Yvette, France). Before each experiment, empty room MEG recordings were acquired for 5 minutes. The experiment consisted of two sessions: 1) 'Rest' (R) session: the subject was asked to keep eyes open and to stare at the black screen in front of him during 5 minutes. 2) 'RDK' session: The subject fixated a cross on the center of the screen, while a random dot kinematogram of $2.5 \mathrm{~s}$ was presented continuously 120 times (radius of the annulus: $4-15 \mathrm{deg}$, dot radius: $0.2 \mathrm{deg}$, dot flow: $16.7 \mathrm{dots}$ per $\operatorname{deg}^{2} . s$, dot speed: $\left.10 \mathrm{deg} / \mathrm{s}\right)$. Initially, all dots were moving incoherently (i.e., each dot moves in a different direction). In half of the trials, $95 \%$ of dots took a coherent direction (ie move in the same direction) after the stimulus onset. In the other half, dots remain incoherent. Moreover, at each frame, $5 \%$ of all dots were reassigned to new positions with new motion directions for incoherent dots.

\subsection{Empirical evidence of long memory}

For each subject, each session and each sensor, we computed Welch's power spectrum estimate derived in Eq. (1). A representative spectrum computed by averaging all radial gradiometers is shown in Fig. 1 in black and dark blue lines for the R and VM sessions, respectively. The presence of scaling or $1 / f$ behaviour clearly appears as a linear slope in this $l o g$-log plot over the $(0.1-3 \mathrm{~Hz})$ frequency range. In addition, this $1 / f$ power spectral density is clearly different from that measured during the empty recording (light blue line in Fig. 1), thus ruling out the hypothesis that the $1 / f$ is simply driven by the electronic device noise. Interestingly, the power of acquisition noise is upper bounded by that measured during brain activity recordings. Finally, $\alpha$ - and $\beta$-band oscillations emerge during the presentation of visual motion stimuli and even more during the rest, while they do not appear in the empty recording.

As a comparison with the previous method, we also estimated the structure fonctions $S^{d}(j, q)$ for $q=2$ only. Structure fonctions at this 2 nd statistical order are indeed equivalent to the power spectrum while estimated using discrete wavelets. $f$ and $j$ are related one another by $f=\frac{3}{4} \frac{f_{s}}{2^{j}}$, where $f_{s}$ is the sampling frequency. The resulting wavelet-based spectrum also named log-scale diagram $\left(\log _{2} S^{L}(j, 2)\right.$ vs. $\left.\log _{2} 2^{j}=j\right)$ averaged over all radial gradiometers is shown in Fig. 1 where green, red and yellow lines represent 
respectively the R, VM and empty recordings. In contrast to Welch's periodogram $\Gamma_{\text {Welch }}(f)$, the $S^{d}(j, 2)$ estimates are less sensitive to oscillation peaks. Discrete wavelets are thus more appropriate to analyse the $1 / f$ behaviour in low frequencies.

This preliminary result confirmed the presence of scaling in the data, which cannot be attributed to the sensor noise. It also shows the advantage of using discrete wavelets for analyzing the $1 / f$ spectrum. Although spectral analysis provides restricted information on the scale invariance properties, it allows us to determine the scale range on which the WLBMF analysis is applied. In the following, we analyse the multifractal properties in the scale range $\left[j_{m}, j_{M}\right]=[9,14]$ (i.e. in the frequency range $0.1-3 \mathrm{~Hz}$ ). The log-scale diagram is actually linear in this part.

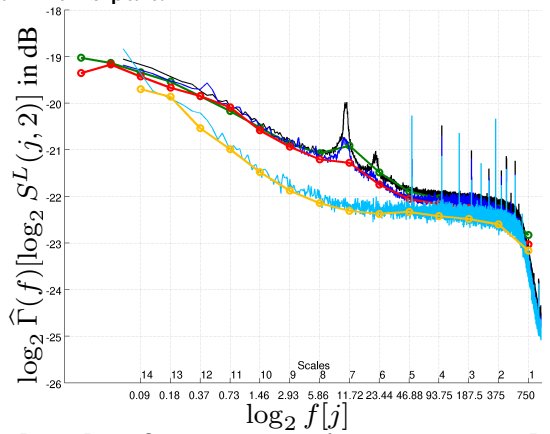

Fig. 1. Log-log plot of spectrum estimates averaged across all radial gradiometers. Welch's periodograms are plotted in black (R), dark blue (VM) and light blue (Empty). Wavelet-based estimates are plotted in green (R), red (VM) and yellow (Empty).

\section{MODULATION OF MULTIFRACTAL PROPERTIES}

\subsection{WLBMF analysis on all sensors}

For each subject $s=1: 20$, considering $N=3$ as the number of vanishing moments of the Daubechies mother wavelet we estimated $\widehat{c}_{1, k, c}^{L, r}(s)$ and $\widehat{c}_{2, k, c}^{L, r}(s)$ on the integrated time series (i.e. the cumulative sum) measured in each sensor $(k=1: 102)$ and for each channel type $c$ : magnetometers (mag) and gradiometers along the radial $\left(\operatorname{grad}_{\mathrm{R}}\right)$ and tangential $\left(\operatorname{grad}_{\mathrm{T}}\right)$ directions. The same estimation was done for the rest $(r=\mathrm{R})$ and visual motion $(r=\mathrm{VM})$ sessions. The mean values $\mu_{i, k, c}^{\mathrm{r}}=\sum_{s=1}^{20} \widehat{c}_{i, k, c}^{L, r}(s) / 20(i=1: 2)$, are plotted for all sensor types in Fig. 2. In both sessions, all sensors exhibit large self-similarity: $\mu_{1, k, c}^{\mathrm{r}}>0.75$. Moreover, the $\mu_{1, k, c}^{\mathrm{r}}$-values appear systematically larger in the frontal regions (meaning more selfsimilar) than in the occipito-parietal ones. Note also that $\mu_{i, k, c}^{\mathrm{r}}>1$ in certain sensors, which violates the validity of $\mathrm{fBm}$ model.

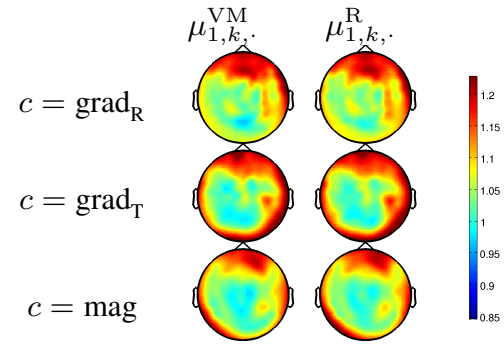

Fig. 2. Group-level $\mu_{1, k, c}^{\mathrm{r}}$-topographies computed for all sensors $k$, all channel types $c$ and the two sessions $r=\mathrm{R}, \mathrm{VM}$.

In the same manner, Fig. 3 shows the topographies of the mean $\mu_{2, k, c}^{\mathrm{r}}$-values. Multifractality $\left(\mu_{2, k, c}^{\mathrm{r}}<0\right)$ is observed on the gradiometers located in the occipito-parietal regions.

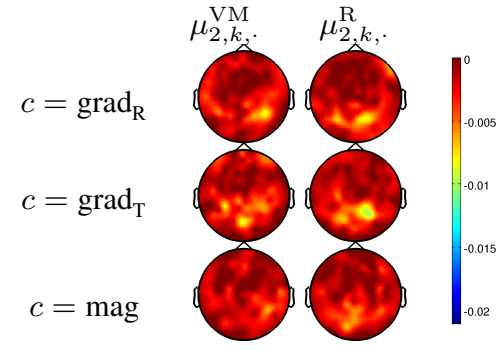

Fig. 3. Group-level $\mu_{2, k, c}^{\mathrm{r}}$-topographies computed for all sensors $k$, all channel types $c$ and the two sessions $r=\mathrm{R}, \mathrm{VM}$.

Then, we perform the following one-sided group-level statistical tests:

$$
\left.\begin{array}{l}
H 0_{(1, k, c)}^{\mathrm{r}}: \mu_{1, k, c}^{\mathrm{r}} \leqslant \delta_{1}, \text { where } \delta_{1}=0.5 \text { (White noise), } \\
H 0_{(2, k, c)}^{\mathrm{r}}: \mu_{2, k, c}^{\mathrm{r}}=\delta_{2}, \text { where } \delta_{2}=0 . \text { (self-similarity), }
\end{array}\right\}
$$

To this end, we computed the $t$-statistics $T_{i, k, c}^{\mathrm{r}}=\left(\mu_{i, k, c}^{\mathrm{r}}-\right.$ $\left.\delta_{i}\right) / \sigma_{i, k, c}^{\mathrm{r}}$, where the latter term denotes the group-level standard deviation. Rejecting $H 0_{(1, k, c)}^{\mathrm{r}}$ clearly amounts to localizing regions $(k)$ and sensor type (c) eliciting self-similarity in session $r$ while rejecting $H 0_{(2, k, c)}^{\mathrm{r}}$ enables to discriminate multifractality from selfsimilarity. Statistical results (not shown) demonstrate that $H 0_{(1, k, c)}^{\mathrm{r}}$ was rejected everywhere even after Bonferroni correction for multiple comparisons ( $\mathrm{p}$-values $p_{\text {corr }}<10^{-6}$ ). However, due to a large between-subject variability, $H 0_{(2, k, c)}^{\mathrm{r}}$ was not rejected after Bonferroni correction ( $p_{\text {uncorr }} \approx 10^{-2}$ ) for a False Positive Rate (FPR) of $5 \%$. Paired $t$-tests were also computed to compare the R and VM sessions, where the null assumption consists in assuming the same mean values: $\mu_{i, k, c}^{\mathrm{R}}=\mu_{i, k, c}^{\mathrm{VM}}$ The null hypotheses $H 0_{(1, k, c)}^{\mathrm{R}, \mathrm{VM}}$ and $H 0_{(2, k, c)}^{\mathrm{R}, \mathrm{VM}}$ were never rejected indicating that no significant difference can be exhibited between ongoing and evoked activity. However, in several MEG studies, the signal of interest is usually considered as a non-linear combination of both types of gradiometers, namely the $\ell_{2}$-norm of gradiometers. Therefore, we decided to analyse the norm of gradiometers too.

\subsection{WLBMF analysis on the norm of gradiometers}

As each pair of gradiometers is orthogonal, their norm is simply defined by $\|\overrightarrow{\operatorname{grad}}\|=\sqrt{\operatorname{grad}_{\mathrm{R}}^{2}+\operatorname{grad}_{\mathrm{T}}^{2}}$. As already done, we estimated $\widehat{c}_{1, k,\|\cdot\|}^{L, r}(s)$ and $\widehat{c}_{2, k,\|\cdot\|}^{L, r}(s)$ for $\|\operatorname{grad}\|$ and then computed mean values $\mu_{i, k,\|\cdot\|}^{\mathrm{r}}(i=1,2)$. A very noticeable result (Fig. 4[left]) is the global reduction of self-similarity shown by the decrease of $\mu_{1, k,\|.\|}^{\mathrm{r}}$ in both sessions. Nonetheless, the self-similarity remains large enough to be statistically significant everywhere. We then compared the two sessions by computing $\Delta \mu_{1, k,\|\cdot\|}=\mu_{1, k,\|\cdot\|}^{\mathrm{R}}-\mu_{1, k,\|\cdot\|}^{\mathrm{VM}}$ (see Fig. 4[Mid.]) and testing the statistical significance of this difference using a paired $t$-test where the null hypothesis was $H 0^{\mathrm{R}, \mathrm{VM}}$ :

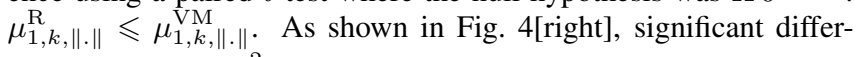
ences $\left(p_{\text {corr }}<10^{-2}\right)$ emerges in the occipital area where $\Delta \mu_{1, k,\|.\|}>$ 0 . This finding is consistent with the literature dealing with the taskinduced modulation of scale-free properties [4] observed in fMRI.

The multifractal behaviour of $\|\overrightarrow{\operatorname{grad}}\|$ measured through negative values of $\mu_{2, k,\|.\|}^{\mathrm{r}}$ is emphasized in Fig. 5. As shown in Fig. 3[Mid.], $\Delta \mu_{2, k,\|.\|}<0$ in the occipito-parietal (MT) area. This means that multifractality decreases in the target region (MT) of the delivered stimuli during evoked activity (cold spots in Fig. 5[Left]) in comparison with resting-state. The statistical paired $t$-test performed with $H 0^{\mathrm{R}, \mathrm{Vm}}: \mu_{2, k,\|\cdot\|}^{\mathrm{R}} \geqslant \mu_{1, k,\|\cdot\|}^{\mathrm{VM}}$ is significant in the MT region but not enough to survive to Bonferroni correction for $\mathrm{FPR}=5 \%$. 

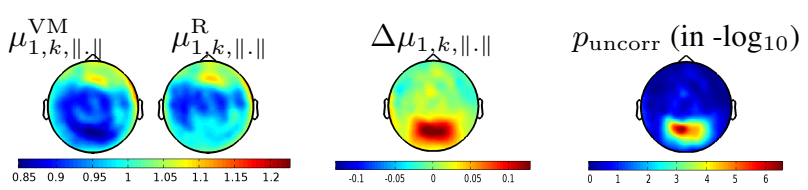

Fig. 4. Left: Group-level $\mu_{1, k, c}^{\mathrm{r}}$-topographies computed for the norm of the gradiometers and the two sessions $r=\mathrm{R}, \mathrm{VM}$. Middle: mean difference $\Delta \mu_{1, k,\|.\|}$ between the R and VM sessions. Right: Uncor. p-values resulting from the between-session paired $t$-test.
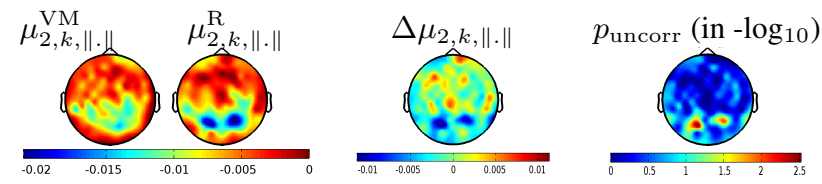

Fig. 5. Left: Group-level $\mu_{2, k, c}^{\mathrm{r}}$-topographies computed for the norm of the gradiometers and the two sessions $r=\mathrm{R}, \mathrm{VM}$. Middle: mean difference $\Delta \mu_{2, k,\|.\|}$ between the R and VM sessions. Right: Uncor. p-values resulting from the between-session paired $t$-test.

\subsection{Simulation with fGn}

To understand the impact of the $\ell_{2}$ norm on the multifractal properties, we synthesized $10000 \mathrm{fGn}$ processes $X(H=.75)$ with the circulant embedded method [13]. We considered a simplified problem where $\operatorname{grad}_{\mathrm{R}}=\operatorname{grad}_{\mathrm{T}}$, meaning that $\|\overrightarrow{\operatorname{grad}}\| \propto \sqrt{\operatorname{grad}_{\mathrm{R}}^{2}}=\left|\operatorname{grad}_{\mathrm{R}}\right|$. Hence, our simulation amounts to estimating the multifractal properties of $X$ and $|X|$. We systematically observed a decrease of long memory: $\Delta \widehat{c}_{1} \triangleq \widehat{c}_{1_{|\mathrm{X}|}}-\widehat{c}_{1_{\mathrm{X}}}<0$, which is consistent with our experimental results. Likewise, multifractality appeared in $|X|$ whereas it is absent in $X$ (fGn process). Given that the number of sign changes $n_{\text {sign }}$ in $X$ is the only parameter that induces regularity changes in $|X|$ (i.e. no addition of irregularity if $X$ is always positive or negative), $n_{\text {sign }}$ was expected to be the main cause of these observations. This hypothesis is validated in Fig. 6 when plotting $\Delta \widehat{c}_{1}$ with respect to the proportion of sign changes $p_{\text {sign }}$ in $X . \Delta \widehat{c}_{1}$ decreases almost systematically (i.e. with a low variance) as a function of $p_{\text {sign }}$. It goes down dramatically when $p_{\text {sign }}>0.3$. Regarding the multifractality (i.e. $\Delta \widehat{c}_{2}$ ), the observation is similar but less pronounced owing to the higher estimator variance (figure not shown).

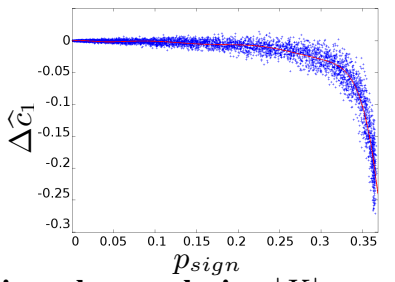

Fig. 6. $\widehat{c}_{1}$ variation when analyzing $|X|$ as a function of $p_{s i g n}$. $10000 \mathrm{fGn}$ processes synthesized (in blue). Fitted curve in red.

Therefore, the apparent contrast in the occipital area when analyzing the norm of gradiometers can be explained by the number of sign changes in the gradiometers or by their phase. Because gradiometers measure the spatial derivative of the magnetic fields in two orthogonal directions, a change of sign suggests a change of source orientation or even perhaps of the source itself.

\section{DISCUSSION AND CONCLUSION}

We have demonstrated the presence of long memory in MEG data over all sensors. A small amount of multifractality was observed on the gradiometers in the occipital and parietal scalp regions. Interestingly, only the norm of gradiometers exhibits a modulation of the multifractal properties between ongoing and evoked activity. Additionally, this modulation was very localized to an area probably sensitive to the nature of the stimuli (as assessed by additional experimental work in progress). It would be very interesting to change the sensory context (e.g. auditory stimuli) in order to check whether the modulation moves to the expected auditory regions in order to further show the specificity of our results.

The norm of gradiometers seems to capture more information. This is largely due to the nonlinear nature of the norm and the number of sign changes in the gradiometer signals. The contrast between $\mathrm{R}$ and $\mathrm{RDK}$ in the occipital area can be interpreted as a higher rate of source orientation changes in hMT+/V5 during the presentation of RDK. Importantly, we propose that this result extends to a more general concept: in any imaging modality (e.g. fMRI), the knowledge of any nonlinear transformation in the generative model [14] of the data can be crucial to correctly interpret its multifractal properties.

\section{REFERENCES}

[1] BJ He, JM Zempel, AZ Snyder, and ME Raichle, "The Temporal Structures and Functional Significance of Scale-free Brain Activity," Neuron, vol. 66, no. 3, pp. 353-, 2010.

[2] TH Bullock, MC Mcclune, and JT Enright, "Are the electroencephalograms mainly rhythmic? Assessment of periodicity in wide-band time series," Neurosci, vol. 121, no. 1, pp. 233-, 2003.

[3] WJ Freeman and J Zhai, "Simulated power spectral density (PSD) of background electrocorticogram (ECoG).," Cognitive neurodynamics, vol. 3, no. 1, pp. 97-, 2009.

[4] BJ He, "Scale-Free Properties of the Functional Magnetic Resonance Imaging Signal during Rest and Task," J. of Neuroscience, vol. 31, no. 39, pp. 13786-, 2011.

[5] P Ciuciu, G Varoquaux, P Abry, and M Almog, "Multifractal analysis of resting state networks in functional MRI," in 8th Proc. IEEE ISBI, Chicago, IL, 2011, pp. 473-.

[6] S Sadaghiani, G Hesselmann, KJ Friston, and A Kleinschmidt, "The relation of ongoing brain activity, evoked neural responses, and cognition.," Front. in syst neurosci, vol. 4, pp. 20, 2010.

[7] D Van de Ville, J Britz, and CM Michel, "EEG microstate sequences in healthy humans at rest reveal scale-free dynamics.," PNAS USA, vol. 107, no. 42, pp. 18179-, 2010.

[8] M Buiatti, D Papo, P-M Baudonnière, and C van Vreeswijk, "Feedback modulates the temporal scale-free dynamics of brain electrical activity in a hypothesis testing task.," Neurosci, vol. 146, no. 3, pp. 1400-, 2007.

[9] J Suckling, AM Wink, F Bernard, A Barnes, and E Bullmore, "Endogenous multifractal brain dynamics are modulated by age, cholinergic blockade and cognitive performance.," J. of neuroscience methods, vol. 174, no. 2, pp. 292-, 2008.

[10] H Wendt, P. Abry, and S Jaffard, "Bootstrap for empirical multifractal analysis,” IEEE Signal Proc. Mag., vol. 24, pp. 38-, 2007.

[11] K Linkenkaer-Hansen, VV Nikouline, JM Palva, and RJ Ilmoniemi, "Long-range temporal correlations and scaling behavior in human brain oscillations.," J. of neuroscience, vol. 21, no. 4, pp. 1370-, 2001.

[12] P Ciuciu, P Abry, C Rabrait, and H Wendt, "Log Wavelet Leaders Cumulant Based Multifractal Analysis of EVI fMRI Time Series: Evidence of Scaling in Ongoing and Evoked Brain Activity," IEEE J. Sel. Top. in Sig. Proc., vol. 2, pp. 929-, 2008.

[13] C Dietrich and G Newsam, "Fast and exact simulation of stationary Gaussian processes through circulant embedding of covariance matrix," SIAM Sci. Comput., vol. 18, pp. 1088-, 1997.

[14] T. Vincent, L. Risser, and P. Ciuciu, "Spatially adaptive mixture modeling for analysis of within-subject fMRI time series," IEEE Trans. Med. Imag., vol. 29, no. 4, pp. 1059-, 2010. 